\title{
The functional integrity and fate of cryopreserved ram spermatozoa in the female tract
}

\author{
L. Gillan and W. M. C. Maxwell \\ Department of Animal Science, University of Sydney, NSW 2006, Australia
}

\begin{abstract}
Cryopreservation advances capacitation-like changes in ram spermatozoa. These changes are reflected in an increased fertilizing ability compared with fresh spermatozoa, followed by an accelerated decline in fertilizing ability after incubation in vitro or in vivo. Furthermore, frozen-thawed spermatozoa are released earlier than fresh spermatozoa after binding to oviduct cells in vitro, confirming their physiological readiness to participate in fertilization despite their short lifespan. After insemination large numbers of spermatozoa are lost from the female reproductive tract of the ewe via the vagina. Frozen-thawed spermatozoa are expelled faster than fresh spermatozoa. The advanced membrane status of frozen-thawed spermatozoa may provoke their rapid loss and possibly makes them more vulnerable to attack by uterine leucocytes, or by some other mechanism, as a high proportion of spermatozoa lost from the tract are decapitated. The observed destabilization of the membranes of cryopreserved spermatozoa is accompanied by impaired sperm transport, associated with mitochondrial injury, necessitating intrauterine deposition of frozen-thawed semen to obtain satisfactory fertility after artificial insemination. However, the frozen-thawed spermatozoa that can participate in fertilization may contribute to increased embryonic loss by the advancement of cleavage or through a direct effect of cryopreservation on the male genome.
\end{abstract}

\section{Introduction}

The discovery of cryoprotective properties of glycerol (Bernstein and Petropavlovskij, 1937) led to the routine use of cryopreserved semen for artificial insemination in cattle. However, its application in the artificial insemination of other livestock is limited, due to reduced fertility after non-surgical insemination compared with that of fresh or liquid-stored semen. This reduced fertility results from factors such as poor recovery of viability after cryopreservation (as in pigs and horses) and reduced sperm transport in the female tract, necessitating costly intrauterine insemination (as in sheep and goats). Although considerable progress has been made in some aspects of gamete and embryo cryopreservation, such as pig embryo vitrification (Dobrinsky and Johnson, 1994), there has been little corresponding advance in boar semen cryopreservation (Almlid and Hofmo, 1996). This is due in part to economic considerations, since liquid semen delivery systems are well advanced in the pig industry and cryopreservation is, therefore, required only for the importation of new genes and the banking of valuable germplasm. However, in other livestock, such as sheep, where a low-cost method for utilization of cryopreserved spermatozoa would be of considerable economic advantage, progress has been limited despite considerable research (Salamon and Maxwell, $1995 \mathrm{a}, \mathrm{b})$.

The purpose of this review is to consider, with particular reference to the ram, recent findings on the effects of cryopreservation on sperm function and the fate of cryopreserved spermatozoa in the female reproductive tract. 


\section{Factors Associated with Poor Fertility of Cryopreserved Ram Spermatozoa}

Although motility is preserved in a relatively high proportion (40-60\%) of ram spermatozoa after freeze-thawing, only 20-30\% remain biologically unchanged (Salamon and Maxwell, 1995a). It is possible that cryopreservation selects the most viable spermatozoa and destroys the rest, leaving a much smaller, but fertile population (Watson, 1995). However, although this theory is supported by the observed improvement in fertility in ewes after cervical insemination with a greater number of frozen-thawed spermatozoa (Salamon, 1977), it does not fully explain the low fertility of frozen semen. Even when very large numbers of motile, frozen-thawed spermatozoa are placed in the cervix, fertility is lower than for fresh semen (Maxwell and Hewitt, 1986). This finding suggests that the spermatozoa regaining motility after thawing must be compromised in some way that prevents them either reaching the site of fertilization or participating in fertilization. Thus, cryogenic changes to spermatozoa must be responsible for a decrease in their functional integrity or effective transport through the female tract to the site of fertilization.

\section{Functional Integrity of Spermatozoa}

Ultrastuctural and biochemical damage occurs to many spermatozoa during freeze-thawing but those remaining motile, and presumably intact, are functional, giving high fertilization rates (85-93\%) after intrauterine or tubal deposition of even very small numbers of spermatozoa (sheep: Maxwell et al., 1993; cattle: Seidel et al., 1995). Although it has been assumed that the functional condition of this motile subpopulation of cryopreserved spermatozoa would be similar to that of fresh motile spermatozoa, evidence suggests that this is not the case, as a greater proportion of the surviving spermatozoa show evidence of altered membrane responses to physiological stimuli (Watson, 1995; Maxwell and Watson, 1996).

\section{Capacitation status of spermatozoa}

Ejaculated spermatozoa cannot fertilize until they undergo capacitation, which is the first stage of membrane destabilization events involving an efflux of cholesterol and redistribution of intrinsic membrane proteins and lipids (Harrison, 1996). This destabilization continues with the acrosome reaction and ultimately results in loss of viability. Cryopreservation suspends the continuum of changes in spermatozoa that begins in the epididymis and ends at fertilization or cell death. However, rather than being revived in the same state as they were before freezing, the spermatozoa may emerge in a state resembling that of capacitation, having bypassed some of this normal maturation (Watson, 1995). Watson proposed that the apparently 'capacitated' cells in frozenthawed semen would have a reduced life-span and if not exposed to oocytes within a short time would be unable to achieve fertilization. Ageing of functionally capacitated ram spermatozoa in the reproductive tract of the ewe after cervical insemination may further aggravate this situation, and such spermatozoa may die prematurely in the lower part of the reproductive tract.

To test Watson's hypothesis, Gillan et al. (1997a) investigated the proposal that cryopreservation may cause ram spermatozoa to undergo membrane changes similar to capacitation. The events of capacitation may involve an influx of calcium ions. The antibiotic chlortetracycline (CTC, a fluorescent compound) accumulates and fluoresces in membrane compartments in which there are high concentrations of calcium ions next to hydrophobic sites (Tsien, 1989). CTC has been used as a fluorescent probe to visualize the course of capacitation and the acrosome reaction in mouse (Saling and Storey, 1979), bull (Fraser et al., 1995) and boar spermatozoa (Wang et al., 1995; Maxwell and Johnson, 1997a). The CTC patterns assessed by Gillan et al. (1997a) were F (non-capacitated), B (capacitated) and AR (acrosome reacted). A high proportion of the spermatozoa had either capacitated or acrosome reacted, as determined by these patterns, after incubation at $37^{\circ} \mathrm{C}$ for $6 \mathrm{~h}$ or after freezing and thawing with or without further incubation, whereas most fresh unincubated spermatozoa had not capacitated (Fig. 1). In vitro matured oocytes were penetrated earlier by frozen 

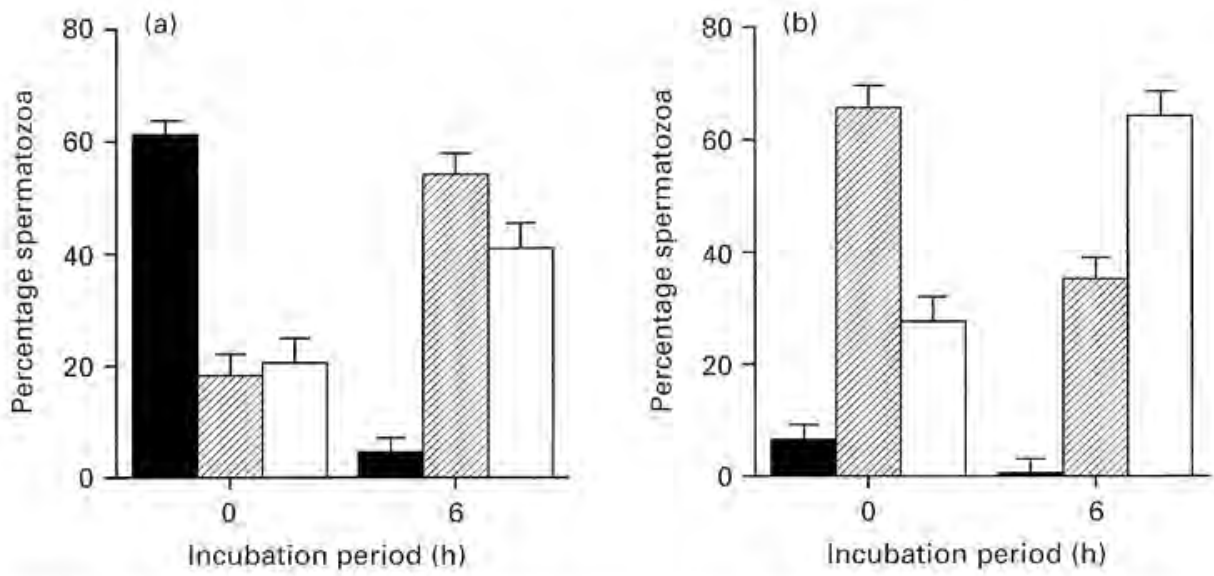

Fig. 1. The distribution of $\mathrm{F}$ (- non-capacitated), B (D; capacitated) and AR ( $\square$; acrosomereacted) chlortetracycline staining patterns at $t=0 \mathrm{~h}$ (no incubation) or $6 \mathrm{~h}$ (incubation at $37^{\circ} \mathrm{C}$ ) for fresh (a) and frozen-thawed (b) ram spermatozoa. Values are means ISEM of six replicates. (Adapted from Gillan et al., 1997a.)

Table 1. Proportion of advanced zygotes (cleavage stage or at syngamy with the sperm tail present) $24 \mathrm{~h}$ after in vitro fertilization of in vitro matured sheep oocytes with fresh and frozen-thawed ram spermatozoa

\begin{tabular}{lcccc}
\hline \multirow{2}{*}{$\begin{array}{l}\text { Type of } \\
\text { spermatozoa }\end{array}$} & $\begin{array}{c}\text { Incubation time } \\
\left.\text { (h at } 37^{\circ} \mathrm{C}\right)\end{array}$ & Zygotes assessed & \multicolumn{2}{c}{ Zygotes advanced } \\
\hline Fresh & 0 & 88 & Number & $\%$ \\
\multirow{2}{*}{ Frozen-thawed } & 6 & 62 & 24 & 27.3 \\
& 0 & 76 & 53 & 67.7 \\
& 6 & 40 & 30 & 75.7 \\
\hline
\end{tabular}

(L. Gillan, G. Evans and W. M. C. Maxwell, unpublished.)

than by fresh spermatozoa, as evidenced by the presence of more advanced zygotes $24 \mathrm{~h}$ after insemination (Table 1). Fresh spermatozoa incubated for $6 \mathrm{~h}$ at $37^{\circ} \mathrm{C}$ produced advanced zygotes in similar proportions to frozen spermatozoa.

These findings suggest that both incubation and freeze-thawing advance capacitation-like changes in ram spermatozoa and that further incubation of such cells causes them to acrosome react. Similar findings have been published for bovine spermatozoa (Cormier et al., 1997). The in vitro fertilization results also indicate that the cryopreserved spermatozoa are capacitated, confirming earlier findings that frozen ram spermatozoa exhibit the highest penetration of zona-free hamster eggs immediately after thawing in contrast to fresh spermatozoa, which require a period of incubation of several hours to attain the highest penetration rate (Watson, 1995).

Other treatments that might be expected to increase the proportions of capacitated spermatozoa in boar semen (incubation in the presence of bicarbonate, flow cytometric sorting, cooling and freezing) also increase the percentage of B-pattern cells in comparison with fresh semen (Maxwell and Johnson, 1997a). Lipid phase transitions have been implicated as responsible for membrane destabilization during cooling (Holt and North, 1984). It is probably the process of cooling and rewarming that induces capacitation-like changes in membrane function after cryopreservation rather than events associated with ice formation, and thus cells merely cooled to $0-4^{\circ} \mathrm{C}$ also display this effect (Watson, 1996; Maxwell and Johnson, 1997a,b). However, it is not possible to draw definitive conclusions from these results about the importance of sperm membrane changes 
associated with frozen storage when the semen is used for artificial insemination. Even after prolonged ageing or freeze-thawing of spermatozoa, the number of acrosome-intact cells in the inseminate may still be above the threshold required to achieve satisfactory fertilization of oocytes.

\section{Integrity of the sperm tail}

In addition to changes in capacitation status of the spermatozoa, the integrity of the sperm tail and its mitochondrial function may also be influenced by freezing and thawing. Windsor (1997) demonstrated that mitochondrial respiration was important for cervical penetration by ram spermatozoa and that mitochondrial injury, as assessed by uptake of rhodamine 123 (R123), was a significant contributor to the poor fertility in ewes after cervical compared with intrauterine insemination with frozen-thawed semen. This effect was independent of post-thaw motility of spermatozoa. The same study demonstrated that inhibition of glycolysis by rotenone did not prevent fertilization of ova by ram spermatozoa, but did reduce their ability to traverse the cervix and reach the site of fertilization. Our studies (L. Gillan, G. Evans and W. M. C. Maxwell, unpublished) suggest that mitochondrial function is influenced by freezing and thawing in a similar way to functional membrane status (as illustrated in Fig. 1). Fresh spermatozoa incubated for $6 \mathrm{~h}$ at $37^{\circ} \mathrm{C}$ exhibited the same uptake of $\mathrm{R} 123$ as frozen-thawed, unincubated spermatozoa (Fig. 2). There was a more rapid decline in the proportion of frozen-thawed than fresh spermatozoa able to take up R123 during incubation $(P<0.001)$, indicating that fresh spermatozoa maintained their respiratory activity longer than frozen-thawed cells.

The fertility observed by Windsor (1997) in ewes inseminated with rotenone-treated semen (low after cervical but high after intrauterine insemination) is similar to that reported with frozen-thawed ram semen (Lightfoot and Salamon, 1970a; Maxwell and Hewitt, 1986). Windsor (1997) also demonstrated that the cryoprotective procedures currently used for ram semen, including those that enhance post-thaw motility, do not prevent injury to the mitochondria of spermatozoa during the freeze-thawing process. Mitochondrial injury, causing impaired sperm transport, and advanced head membrane destabilization may result in the premature death of large numbers of frozenthawed spermatozoa in the lower parts of the female tract after cervical or vaginal deposition.

\section{Integrity of the sperm genome and embryonic survival}

Early embryonic mortality may contribute to the low fertility rate after artificial insemination of ewes with cryopreserved semen (Salamon and Maxwell, 1995b). An increase in abnormalities of embryonic development associated with ageing of spermatozoa has been observed for several species, and considerable attention has been focused on possible changes in the haploid genome of the sperm cell due to ageing. There is some evidence that embryonic loss may be increased when stored spermatozoa are further aged in the female tract resulting in asynchrony between the age of the spermatozoa and ova (Salamon et al., 1979). Thus, there was a steeper decline in embryonic survival with time of chilled $\left(5^{\circ} \mathrm{C}\right)$ storage of ram semen after single than after double insemination, pointing to the importance of time of semen deposition into the cervix to avoid additional ageing of spermatozoa.

A direct effect of freeze-thawing on the sperm nucleus cannot be ruled out, as cryopreservation is known to cause some DNA denaturation, as assessed by the uptake of acridine orange, of human (Royere et al., 1988) and ram spermatozoa (L. Gillan and W. M. C. Maxwell, unpublished; Fig. 3). Spermatozoa weakened or not totally injured or capacitated during freeze-thawing, and those which after thawing and insemination are aged in the female tract may initiate embryos that are not viable and perish at an early stage. The advanced stage of fertilization obtained after in vitro fertilization of in vitro matured oocytes with frozen spermatozoa (Table 1) may not have resulted in normal embryonic development. The fast cleavage observed also after cervical insemination with liquid stored semen (Lopyrin and Rabocev, 1968) can be considered to presage embryonic mortality. There is some evidence (Gillan et al., 1997) that ewes inseminated into the uterine horns with 


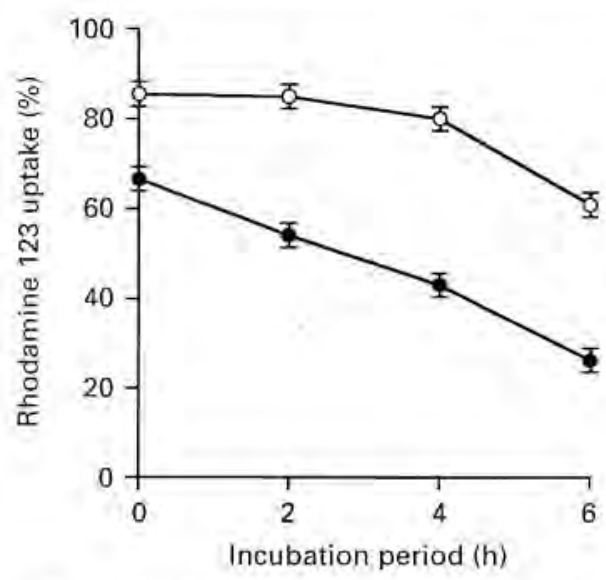

Fig. 2. Uptake of rhodamine 123 by fresh ( 0 ) and frozen-thawed ram spermatozoa (-) after incubation at $37^{\circ} \mathrm{C}$ in air at a concentration of $100 \times 10^{-6} \mathrm{ml}^{-1}$. Values are means \pm SEM of three replicates. (L. Gillan, G. Evans and W. M. C. Maxwell, unpublished.)

frozen-thawed spermatozoa, and at a time closely associated with the expected presence of an oocyte(s), are more likely to produce viable embryos.

\section{Transport of Spermatozoa Through the Female Tract}

The initial theory of sperm transport in sheep and cattle was outlined by Hawk (1983). After semen deposition in the anterior vagina, or in the cervix, spermatozoa were thought to travel to the oviducts in two phases - an initial rapid phase in which small numbers of spermatozoa reached the oviducts within a few minutes, followed by a slower ascent of spermatozoa from cervical reservoirs into and through the uterus, with a gradual increase over several hours of numbers of spermatozoa in the oviducts. It was established by Hunter et al. (1980), using ligation of segments of the oviduct to prevent post-mortem effects on sperm transport, that viable spermatozoa did not reach the oviducts until several hours after mating. Hunter showed that the main functional sperm reservoir in sheep was the caudal portion of the isthmus, and that spermatozoa did not enter the ampulla of the oviduct until after ovulation (Hunter and Nichol, 1983).

A number of investigators (reviewed by Salamon and Maxwell, 1995b) have reported inadequate transport and reduced viability of frozen-thawed spermatozoa in the reproductive tract of the ewe, which contributed to low fertility after cervical insemination. When the thawed semen was deposited into the uterus or oviducts, high (85-95\%) fertilization rates were obtained, indicating that the spermatozoa remaining biologically intact maintained their fertilizing capacity after freeze-thawing. Nevertheless, sperm transport may be impaired even after uterine insemination of cryopreserved semen; thus, fertilization failure in superovulated ewes after late insemination with frozen-thawed spermatozoa was associated with the inability of spermatozoa to pass through the uterotubal junction and isthmus (Jabbour and Evans, 1991). These findings support the original contention of Lightfoot and Salamon (1970a) that an impairment of sperm transport through the female reproductive tract is a major contributor to poor fertility after artificial insemination of sheep with cryopreserved semen. The reduced viability of frozen-thawed spermatozoa in the female reproductive tract is characterized by longevity, which is often half that of fresh spermatozoa (reviewed by Salamon and Maxwell, 1995b) and impaired ability to penetrate the cervix (Lopyrin 


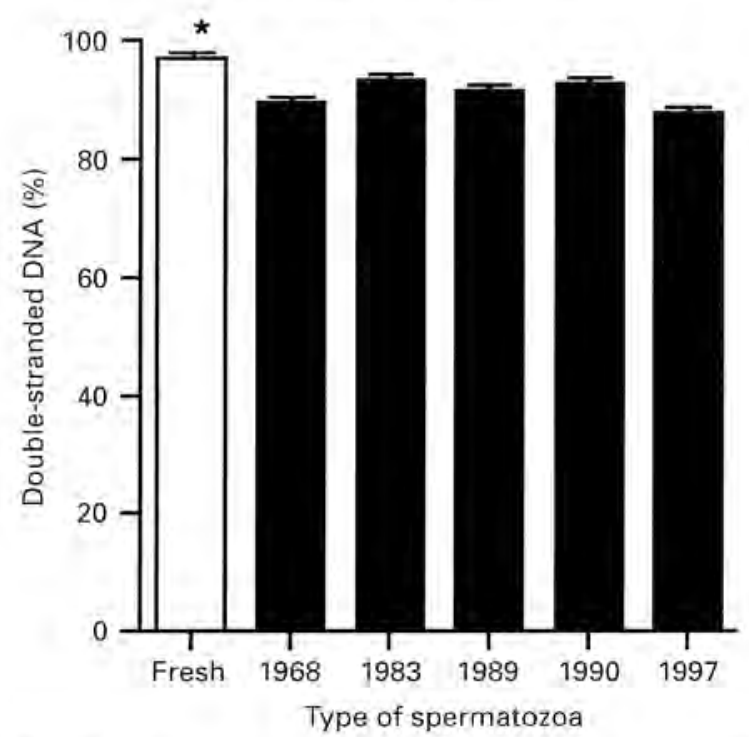

Fig. 3. Percentage of double-stranded DNA, as assessed by uptake of the metachromatic dye acridine orange, in fresh (G) ram spermatozoa compared with spermatozoa frozen ( $\mathbf{D}$ ) in different years. Values are means \pm SEM of three replicates. *Significantly different from values for frozen spermatozoa $(P<0.001)$. (L. Gillan and W. M. C. Maxwell, unpublished.)

and Loginova, 1958), utero-tubal junction or both structures (Mattner et al., 1969; Platov, 1983). However, this may be exacerbated by an increased rate of expulsion of such spermatozoa from the tract. It is not known how many spermatozoa, and in what condition, are required to achieve fertilization, what is the fate of spermatozoa deposited in various parts of the tract and how they interact with epithelial cells when they reach the oviducts.

\section{Number of spermatozoa required to achieve fertility}

The ultimate purpose of sperm transport is to provide adequate numbers of functional spermatozoa, from a heterologous population, at the site of fertilization over a period that will ensure fertilization of all oocytes. Therefore, sperm transport mechanisms need to be both facilitative and regulatory. Given optimal timing of insemination, most species produce more spermatozoa than are required to achieve this response. Consequently, satisfactory fertility can be achieved by artificial insemination of considerably fewer spermatozoa than are in an ejaculate, and the number that reach the site of fertilization at the appropriate time is extremely small compared with the number deposited. However, there are species differences. For example, small numbers of viable spermatozoa are required to achieve fertilization in cows, while pigs require many millions. This quantitative difference may be an important determinant of the fertility of cryopreserved semen, as those species that require large numbers of spermatozoa for conception are less tolerant of poor survival of spermatozoa after cryopreservation.

For achieving comparable fertility rates with fresh and frozen bull spermatozoa, ten times the number of cryopreserved spermatozoa are required (Shannon, 1978). The situation is similar for artificial insemination of sheep, in which a minimum of 100 million viable fresh spermatozoa are required to be deposited in the cervix for adequate fertility. Although cervical insemination of cryopreserved semen is not recommended because of low and variable fertility (Evans and Maxwell, 
1987), published estimates range from 160 (Lightfoot and Salamon, 1970b) to 800 million motile frozen-thawed spermatozoa (Colas, 1979; near the 10:1 ratio for cattle) required to achieve fertility similar to that of fresh semen. However, if in the ewe the cervical barrier to the ascent of spermatozoa is bypassed by surgical or laparoscopic insemination, as few as 100000 or 10000 fresh and 500000 or 100000 frozen-thawed spermatozoa are sufficient to achieve fertilization after insemination into the uterus or oviducts, respectively (Maxwell et al., 1993). These results further support the hypothesis that a combination of impaired transport and reduced functional integrity of spermatozoa are important limiting factors to improved fertility of sheep after artificial insemination with frozen semen.

\section{Fate of spermatozoa deposited in the female tract}

Loss of spermatozoa occurs at all levels of the reproductive tract including the cervix, uterotubal junction and oviducts (reviewed by Drobnis and Overstreet, 1992). The Iow ratio of spermatozoa to ova (approximately 1:1) in the ampulla at the time of fertilization (sheep: Hunter and Nicol, 1986) indicates that these spermatozoa need to be very fertile. For this fertility to be achieved, the female tract must have highly selective mechanisms to eliminate unsuitable cells, and these may also act preferentially to eliminate many cryopreserved spermatozoa before they can reach the site of fertilization.

The fate of spermatozoa that fail to reach the oviducts after natural mating or artificial insemination has been the subject of speculation for many years. In early reports, only a small proportion of the spermatozoa inseminated into the ewe cervix could be recovered (Quinlivan and Robinson, 1969). Hawk and Conley (1971) reported that most spermatozoa in an inseminate drain from the female reproductive tract within a few minutes or hours after insemination; they suggested that the remaining spermatozoa were removed from the tract by slower drainage or phagocytosis. These authors placed a ligature under the mucosa at the vulvo-vaginal junction at artificial insemination to prevent the loss of spermatozoa to the exterior. Twenty-four hours later, $62 \%$ of the spermatozoa in the inseminate were recovered from the ligated reproductive tract. Many of the missing spermatozoa had been removed by phagocytosis, as evidenced by their tails protruding from leucocytes. In contrast, only $0.5 \%$ of the inseminate was recovered from unligated control ewes. Dead spermatozoa deposited in the vagina are known to be lost in large numbers between 3 and $9 \mathrm{~h}$ after insemination (Tilbrook and Pearce, 1984), but it is not clear whether there is a selective mechanism to remove such non-viable compared with viable cells.

Frozen-thawed spermatozoa need to be deposited in the uterus to achieve adequate fertility after artificial insemination of sheep. There have been few reports on the movement of these spermatozoa from the site of insemination and the number of spermatozoa retained in the tract. It is not certain that insemination into the uterus eliminates the cervix as a critical segment of the tract for sperm transport. In an experiment on intrauterine insemination of oestrous ewes $(n=12)$, only $8.0 \pm 5.75 \%$ of fresh and $1.5 \pm 0.89 \%$ of frozen-thawed ram spermatozoa were recovered from the cervix, uterus and oviducts after deposition in the uterine horns by laparoscopy (L. Gillan, P. F, Watson and W. M. C. Maxwell, unpublished). Overall, more spermatozoa were recovered from the uterus $(33.1 \pm 5.69 \%)$ than from the ampulla $(4.0 \pm 3,74 \%)$ or isthmus $(2.9 \pm 5.69 \%$ of the total cells recovered). The low recovery rate reflects considerable loss of cells by phagocytosis or transport out of the reproductive tract. Large numbers of spermatozoa were located in the cervix of the ewes $(67.7 \pm 16.16 \%)$, and many of these cells were motile, indicating a possible role for the cervix as a sperm reservoir after intrauterine insemination with fresh or frozen semen. However, it was not clear whether such cells could have subsequently ascended to the oviducts and participated in fertilization or whether they remained in the lower part of the reproductive tract until death or expulsion through the vagina. This study, together with that of Hawk and Conley (1971), confirms that a high proportion of the inseminate is normally lost through the vagina to the exterior regardless of the site of semen deposition.

Since a large proportion of frozen-thawed spermatozoa may be capacitated, it is likely that the fresh and frozen-thawed spermatozoa would interact in a different way with the female 

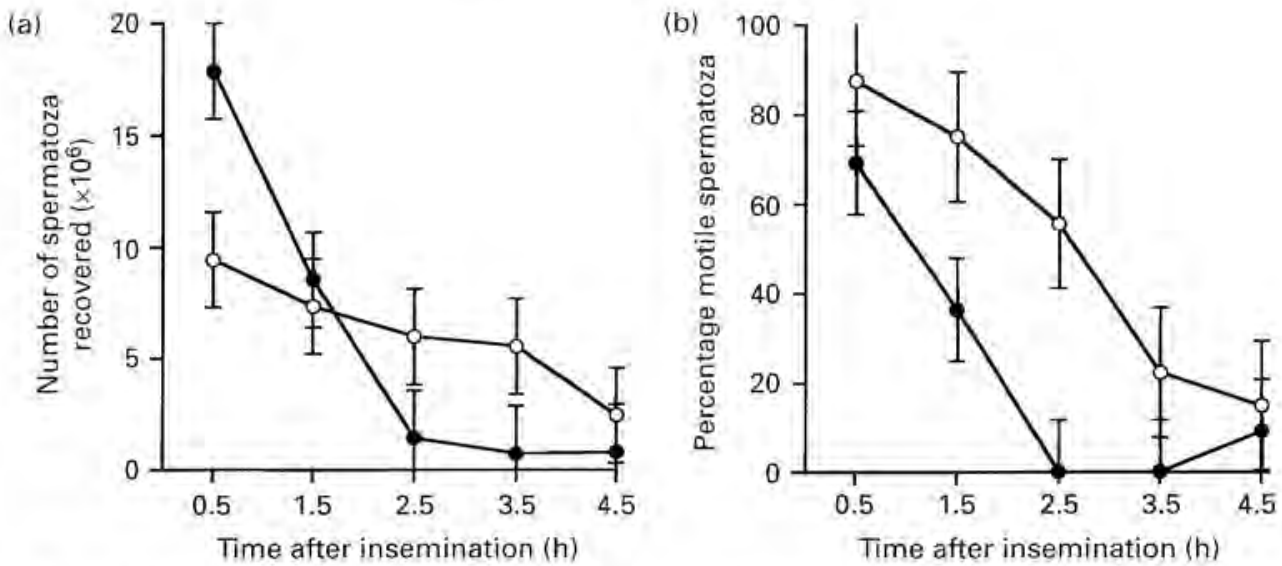

Fig. 4. Number (a) and motility (b) of spermatozoa recovered from the vaginae of ewes after intrauterine insemination with $100 \times 10^{5}$ fresh $(0)$ and frozen-thawed $(\bullet)$ spermatozoa. Values are means \pm SEM of 13 animals, (L. Gillan, P. F. Watson, K. Skogvold and W. M. C. Maxwell, unpublished.)

reproductive tract. Moreover, the frozen-thawed spermatozoa may acrosome-react and die earlier, decreasing the fertilizing population and promoting their loss from the reproductive tract. The rate of loss of spermatozoa from ewes in synchronized oestrus was therefore examined after intrauterine insemination by laparoscopy with $100 \times 10^{6}$ motile Percoll-separated fresh or frozen-thawed spermatozoa (L. Gillan, P. F. Watson, K. Skogvold and W. M. C. Maxwell, unpublished). At the time of insemination, $48 \mathrm{~h}$ after removal of progestagen sponges and injection of 400 i.u. PMSG, a balloon catheter was inserted into the vagina and inflated to prevent loss of spermatozoa. Spermatozoa were recovered from the vagina by flushing the catheter with phosphate-buffered saline after $30 \mathrm{~min}$ and then at hourly intervals after insemination. The overall number of spermatozoa recovered decreased from $13.63 \pm 0.31 \times 10^{6}$ after $0.5 \mathrm{~h}$ to $1.64 \pm 0.36 \times 10^{6}$ at $4.5 \mathrm{~h}$ after insemination $(P<0.001)$. Over the entire recovery period ( $4.5 \mathrm{~h}$ after insemination), equal numbers of fresh and frozen-thawed spermatozoa were recovered from the tract. However, fresh spermatozoa were lost gradually from the tract, whereas large numbers of frozen-thawed spermatozoa were lost within $0.5 \mathrm{~h}$ of insemination and these losses decreased as time within the tract increased $(P<0.05$, Fig. 4 ).

Spermatozoa recovered from the tract were stained with CTC (see Fig. 5). As time within the tract increased, the proportion of $\mathrm{F}$ and $\mathrm{B}$ pattern spermatozoa decreased ( $P<0.001$ in each case) and $A R$ pattern increased. However, there were more AR pattern cells recovered when frozen-thawed spermatozoa were inseminated $(P<0.001)$. More motile fresh than frozen-thawed spermatozoa were recovered $(P<0.05)$ and as the collection period increased the overall motility decreased $(P<0.001$, Fig. 4$)$.

Phagocytosed spermatozoa are often seen in flushings of the reproductive tract (Hawk, 1987), and most of the spermatozoa not lost by drainage or expulsion to the exterior are probably ultimately phagocytosed. However, significant numbers of decapitated or 'tailless' spermatozoa are found in the female reproductive tract after insemination of ewes with both fresh (Mattner, 1964) and frozen spermatozoa (Hawk and Conley, 1971). In the experiment described above, large numbers of decapitated spermatozoa were recovered from the tract $(50.3 \% \pm 0.95$ decapitated compared with $<1 \%$ before insemination), with significantly more frozen-thawed than fresh spermatozoa lacking tails $(P<0.05)$. Moreover, in an earlier experiment (L. Gillan, P. F. Watson and W. M. C. Maxwell, unpublished), more decapitated spermatozoa were found in the cervix $(31.1 \pm 7.01 \%)$, uterus $(42.4 \pm 1.77 \%)$ and isthmus $(32.3 \pm 1.66 \%)$ than in the ampulla $(10.55 \pm 1.94 \%)$. These results indicate that there may be another important, but as yet unknown, mechanism for removal of the tails from 
(a)

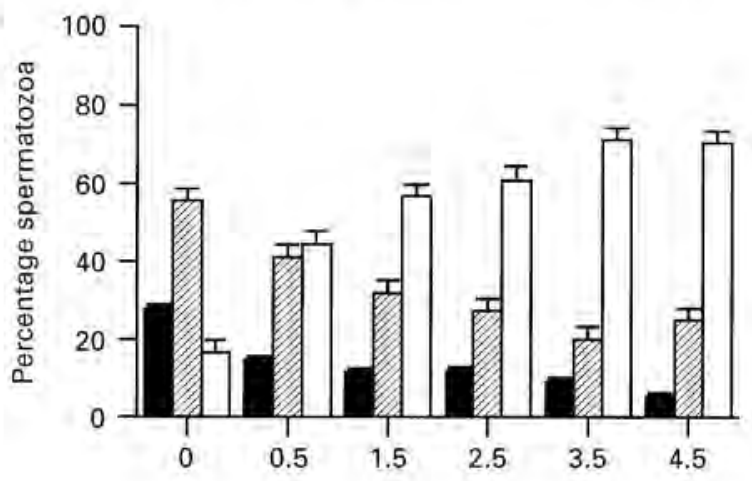

(b)

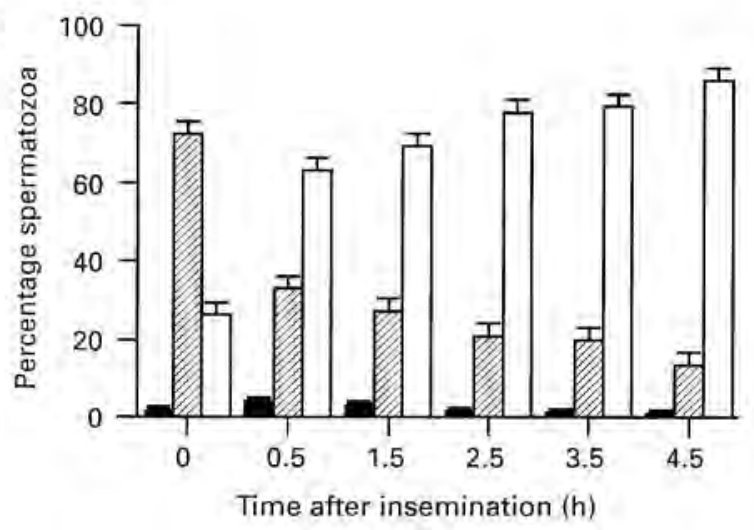

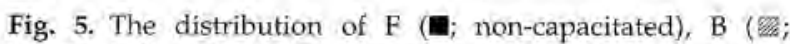
capacitated) and AR ( $\square$; acrosome reacted) chlortetracycline staining patterns of fresh (a) and frozen-thawed (b) ram spermatozoa lost from the tract after intrauterine insemination by laparoscopy. The $t=0 \mathrm{~h}$ sample was analysed before insemination. Values are means \pm SEM of 12 animals.

non-viable spermatozoa in the lower and medium regions of the female reproductive tract, thus ensuring that such cells are unable to participate in the fertilization of oocytes.

\section{Interaction of spermatozoa with the oviducts}

In all animal models studied so far, the isthmus of the oviduct acts as a reservoir and conserves the function of spermatozoa if mating or insemination takes place before ovulation. A minimum of $6-8 \mathrm{~h}$ is required for a functional population of spermatozoa to be established in the oviducts of sheep and cows mated at oestrus (Hunter and Wilmut, 1984; Hunter and Nicol, 1986). Immediately before ovulation the oviduct activates a proportion of these spermatozoa to progress to the site of fertilization in the ampulla (Hunter and Nicol, 1983).

The oviduct may also regulate the speed of capacitation of spermatozoa. For example, when hamster spermatozoa were recovered from the isthmus in animals mated shortly after the onset of oestrus, they required additional time in vitro before they could penetrate eggs, but spermatozoa recovered from females immediately after ovulation could penetrate eggs within $30 \mathrm{~min}$ (Smith and Yanagimachi, 1989). Thus, capacitation and activation of cells sequestered in the isthmic reservoir 

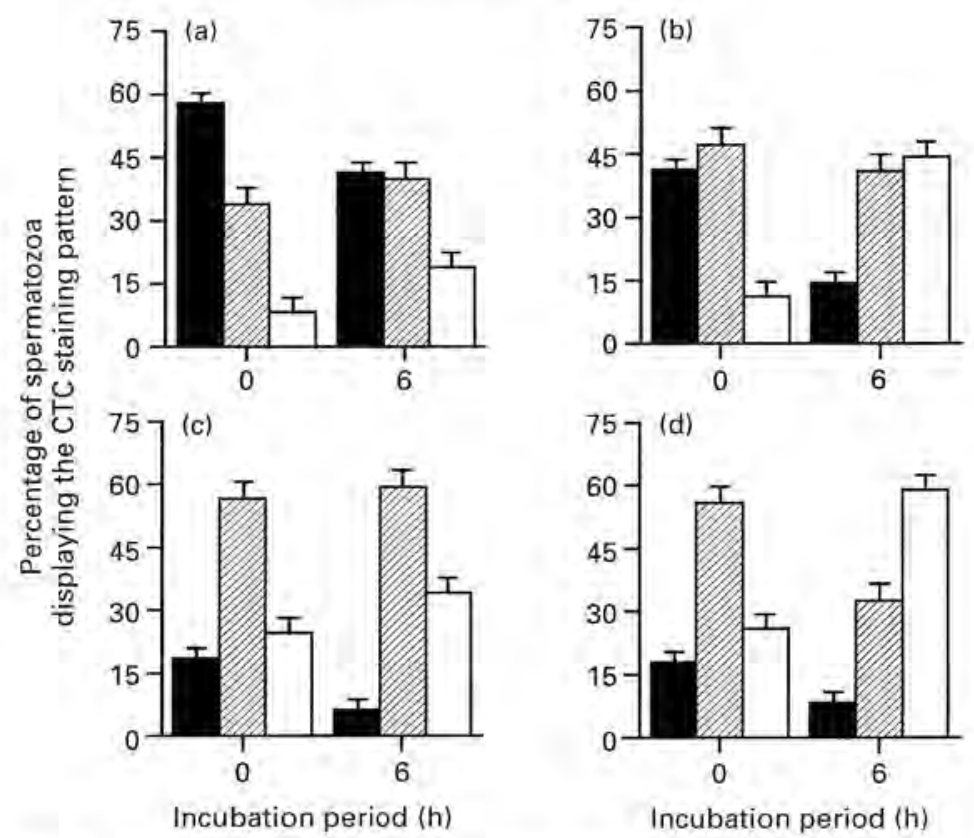

Fig. 6. The percentage of fresh ( $a$ and $b$ ) and frozen-thawed ( $c$ and $d)$

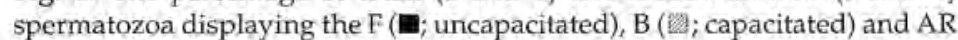
( $\square$; acrosome reacted) chlortetracycline staining patterns after incubation in the absence (control; $a$ and c) or presence (experimental; $b$ and d) of an oviduct epithelial cell monolayer. Values are means \pm SEM of five replicates. (Adapted from Gillan et al., 1997b.)

may not be achieved synchronously but rather be staggered over a period of time (Hunter, 1993). Periods of highly active free swimming appear to be alternated with phases of adhesion to the epithelium. Most uncapacitated hamster spermatozoa deposited in the oviducts attach to the oviductal mucosa, unlike capacitated spermatozoa which do not, indicating that the surface of the spermatozoa regulates these events (Smith and Yanagimachi, 1991). Thus in hamsters, it would appear that spermatozoa remain attached to the isthmic mucosa until they become capacitated, after which they detach and migrate to the ampulla to fertilize the ova.

It is clear that spermatozoa undergo necessary membrane changes during intimate contact with the epithelial cells of the oviduct. As frozen-thawed spermatozoa are likely to be in a more advanced membrane state than freshly ejaculated spermatozoa, these two populations of cells may respond differently when in contact with epithelial cells of the oviduct. Suzuki et al. (1997) found that frozen-thawed bull spermatozoa did react in a different way from fresh spermatozoa when coincubated with bovine oviduct epithelial cells, but they did not assess the membrane status of the spermatozoa. To investigate this possibility further, Gillan et al. (1997b) incubated fresh and frozen-thawed ram spermatozoa in vitro with an oviduct epithelial cell monolayer (OECM) for $6 \mathrm{~h}$, and observed the ability of spermatozoa to attach to the OECM and undergo membrane changes. Control fresh and frozen spermatozoa were incubated in the absence of an OECM. Frozen spermatozoa bound to the OECM immediately and in larger quantities than did fresh spermatozoa. After the removal of unbound spermatozoa, fresh spermatozoa were released from the OECM gradually $(59 \pm 1.0,49 \pm 1.0$ and $27 \pm 2.0 \%$ ), whereas frozen-thawed cells were released rapidly $(58 \pm 3.7,16 \pm 1.9$ and $9 \pm 1.0 \%$ attached after $2 \times 4$ and $6 \mathrm{~h}$ incubation, respectively). A sample of medium containing unattached spermatozoa was also removed to assess membrane status using the CTC fluorescence assay; the majority of the fresh control spermatozoa were initially non-capacitated 
and over the incubation period gradually became capacitated and acrosome reacted (Fig. 6a). In contrast to the control spermatozoa, fresh spermatozoa incubated with OECM were more likely to be capacitated, but acrosome intact at $0 \mathrm{~h}$ (Fig. 6b), perhaps due to the immediate binding of many uncapacitated cells to the OECM. The spermatozoa released from the OECM were mainly capacitated $(51.2 \pm 3.9 \%)$ at $2 \mathrm{~h}$ and most were acrosome reacted at the end of the incubation (Fig. $6 \mathrm{~b}$ ). Most of the frozen control and experimental spermatozoa displayed the B pattern immediately upon thawing (Fig. 6c,d). The control spermatozoa gradually became acrosome reacted (Fig. 6c), whereas the experimental cells underwent this transition more rapidly and to a greater extent (Fig. 6d).

The earlier release of cryopreserved spermatozoa from the OECM may indicate that these cells reached the correct physiological state to participate in fertilization earlier than fresh spermatozoa, adding support to the hypothesis that frozen-thawed spermatozoa are already in a state of membrane destabilization similar to capacitation. The observation that cryopreserved spermatozoa underwent a transition to the acrosome-reacted state earlier than fresh spermatozoa, and earlier in the presence of the OECM than in its absence, indicates that the two types of spermatozoa respond differently to oviduct stimuli and that they have a different lifespan in the female reproductive tract. Thus, even when intrauterine insemination is used to ensure fertilization, mistiming of semen deposition with respect to ovulation will lead to lower fertility than insemination just before, or after, ovulation (Salamon and Maxwell, 1995b). This may be the result of premature exhaustion of the limited supplies of 'uncapacitated' cells from the isthmic reservoirs as a result of their early activation.

\section{Conclusion}

The studies discussed in this paper demonstrate that fresh and frozen-thawed spermatozoa behave differently when deposited in the female reproductive tract. In comparison with fresh spermatozoa, the frozen-thawed cells exhibit destabilized membranes as assessed by CTC fluorescence patterns, capacitation, impaired sperm transport associated with decreased respiratory activity, increased denaturation of DNA, faster loss from the tract and accelerated binding to and release from oviduct cells. These findings support the hypotheses that cryopreservation bypasses the normal processes of sperm maturation leading to capacitation (Watson, 1995) and results in the transport of fewer spermatozoa to the site of fertilization (Lightfoot and Salamon, 1970a).

Studies to date have not explained the mechanisms of the membrane changes observed after cryopreservation or provided strong clues on how they might be reversed. Detailed information is not yet available on the interaction of cryopreserved spermatozoa with the cells within the functional sperm reservoir in the caudal isthmus. However, it is likely that cryopreservation or thawing methods that incorporate strategies to stabilize sperm membranes and limit the rate of loss of spermatozoa from the tract would also ensure that more fertile cells, in the best functional state, reach and establish themselves in the tubal reservoirs.

The authors are grateful to G. Evans, S. Mortimer, S. Salamon and P.F. Watson for their comments on the manuscript.

\section{References}

Almlid T and Hofmo PO (1996) A brief review of frozen semen application under Norwegian AI service conditions. In Proceedings of the Thitd International Conference on Bar Semen Preservation, Mariensee, Germany, Eds D Rath, LA Johnson and KF Weitze Reproduction in Domestic Animals 31 169-174

Bernstein AD and Petropavlovskij VV (1937) Effect of nonelectrolytes on viability of spermatozoa Bjulleten Experimentalnof Biologit i Mediciny 3 41-43 (in Russian)

Colas G (1979) Fertility in the ewe after artificial insemination with fresh and frozen semen at the induced oestrus, and influence of the photoperiod on the semen quality of the ram Livestock Production Science 6 153-166

Cormier N, Sirard M-A and Baley JL (1997) Premature capacitation of bovine spermatozoa is initiated by cryopreservation Journal of Andrology 18 461-468

Dobrinsky JR and Johnson LA (1994) Cryopreservation of porcine embryos by vitrification: a study of in vilro development Theriogenology 42 25-35 
Drobnis EZ and Overstreet JW (1992) Natural history of mammalian spermatozoa in the female reproductive tract Oxford Reviews of Reproductive Biology 14 1-45

Evans G and Maxwell WMC (1987) Salamon's Artificial Insemination of Sheep afid Goats Butterworths; Sydney

Fraser LR, Abeydeera LR and Niwa K (1995) $\mathrm{Ca}^{2+}$-regulating mechanisms that modulate bull sperm capacitation and acrosomal exocytosis as determined by chlortetracycline analysis Molecular Reproduction and Development 40 233-241

Gillan L, Evans G and Maxwell WMC (1997a) The capacitation status and fertility of fresh and frozen-thawed ram spermatozoa Reproduction, Fertility and Development 9 481-487

Gillan L, Evans G and Maxwell WMC (1997b) Cryopreservation induced membrane changes in ram spermatozoa and their effect on oviductal interactions Proceedings of the Australian Society for Reproductioe Biology, Canberra 2885 (Abstract)

Gómez MC, Catt JW, Gillan L, Evans G and Maxwell WMC (1997) Effect of culture, incubation and acrosome reaction of fresh and frozen-thawed ram spermatozoa for in vitro fertilization and intracytoplasmic sperm injection Reproduction, Fertility and Development $9665-673$

Harrison RAP (1996) Capacitation mechanisms, and the role of capacitation as seen in eutherian mammals Reproduction, Fertility and Development 8 581-594

Hawk HW (1983) Sperm survival and transport in the female reproductive tract Journal of Dairy Science 66 2645-2660

Hawk HW (1987) Transport and fate of spermatozoa after insemination of cattle Journal of Dairy Science 70 1487-1503

Hawk HW and Conley HH (1971) Loss of spermatozoa from the reproductive tract of the ewe and intensification of sperm 'breakage' by progestagen Journal of Reproduction and Fertility 27 339-347

Holt WV and North RD (1984) Partially irreversible coldinduced lipid phase transitions in mammalian sperm plasma membrane domains: freeze-fracture study foumal of Experimental Zoology $230473-483$

Hunter RHF (1993) Sperm:egg ratios and putative molecular signals to modulate gamete interactions in polytocous mammals Molecular Reproduction and Development 35 $324-327$

Hunter RHF and Nichol R (1983) Transport of spermatozoa in the sheep oviduct; preovulatory sequestering of cells in the caudal isthmus Journal of Experimental Zoology 228 $121-128$

Hunter RHF and Nichol R (1986) Post-ovulatory progression of viable spermatozoa in the sheep oviduct, and the influence of multiple mating on their pre-ovulatory distribution British Veterinary Journal 142 52-58

Hunter RHF and Wilmut I (1984) Sperm transport in the cow: peri-ovulatory redistribution of cells within the oviduct Reproduction, Nutrition Development 24 597-608

Hunter RHF, Nichol R and Crabtree SM (1980) Transport of spermatozoa in the ewe: timing of the establishment of a functional population in the oviduct Reproduction, Nutrition Development $201869-1875$

Jabbour HN and Evans G (1991) Fertility of superovulated ewes following intrauterine or oviducal insemination with fresh or frozen-thawed semen Reproduction. Fertility and Development 3 1-7

Lightfoot RJ and Salamon S (1970a) Fertility of ram spermatozoa frozen by the pellet method, I. Transport and viability of spermatozoa within the genital tract of the ewe Journal of Reproduction and Fertility 22 385-398
Lightfoot RJ and Salamon S (1970b) Fertility of ram spermatozoa frozen by the pellet method, II. The effects of method of insemination on fertilization and embryonic mortality Journal of Reproduction and Fertility $22.399-408$

Lopyrin AI and Loginova NV (1958) Method of freezing ram semen Ovtsevodstoo No. 831-34 (in Russian)

Lopyrin AI and Rabocev VK (1968) The optimum time to use preserved semen Ovisevodstoo 13 No. 9 34-36 (in Russian)

Mattner PE (1964) Studies on Transport and Distribution of Spermatozoa in the Genital Tract of the Ewe MVSc thesis, University of Sydney

Mattner PE, Entwistle KW and Martin ICA (1969) Passage, survival and fertility of deep-frozen ram semen in the genital tract of the ewe Australian Iournal of Biological Science 22 181-187

Maxwell WMC and Hewitt LJ (1986) A comparison of vaginal, cervical and intrauterine insemination of sheep Journal of Agricultural Science 106 191-193

Maxwell WMC and Johnson LA (1997a) Chlortetracycline analysis of boar spermatozoa after incubation, flow cytometric sorting, cooling, or cryopreservation Molecular Reproduction and Development $46408-418$

Maxwell WMC and Johnson LA (1997b) Membrane status of boar spermatozoa after cooling or cryopreservation Theriogenology 48 209-219

Maxwell WMC and Watson PF (1996) Recent progress in the preservation of ram semen Animal Reproduction 5 cience 42 $55-65$

Maxwell WMC, Evans G, Rhodes SL, Hillard MA and Bindon BM (1993) Fertility of superovulated ewes after intrauterine or oviducal insemination with low numbers of fresh or frozen-thawed spermatozoa Reproduction, Ferfility and Development 557-63

Platov EM (1983) An important factor affecting ewe fertility Ovtsevodstzo No. $635-37$ (in Russian)

Quinlivan TD and Robinson TJ (1969) Number of spermatozoa in the genital tract after artificial insemination of progestagen-treated ewes Journal of Reproduction and Fertility 19 73-86

Royere D, Hamamah S, Nicolle JC, Barthelemy C and Lansac J (1988) Freezing and thawing alter chromatin stability of ejaculated human spermatozoa: fluorescence acridine orange staining and feulgen-DNA cytophotometric studies Ganete Research 21 51-57

Salamon S (1977) Fertility following deposition of equal numbers of frozen-thawed ram spermatozoa by single and double insemination Australian lournal of Agricultural Research 28 477-479

Salamon S and Maxwell WMC (1995a) Frozen storage of ram semen. 1. Processing, freezing, thawing and fertility after cervical insemination Animal Reproduction Science 37 $185-249$

Salamon S and Maxwell WMC (1995b) Frozen storage of ram semen. II. Causes of low fertility after cervical insemination and methods of improvement Animal Reprodiction Science 38 1-36

Salamon S, Maxwell WMC and Firth JH (1979) Fertility of ram semen after storage at $5^{\circ} \mathrm{C}$ Animal Reproduction Science 2 373-385

Saling PM and Storey BT (1979) Mouse gamete interactions during fertilization in vitros chlortetracycline as a fluorescent probe for the mouse sperm acrosome reaction Journal of Cell Biology 83 544-555

Seidel GE, Allen CH, Brink Z, Graham JK and Cattell MB (1995) Insemination of Holstein heifers with very low numbers of 
unfrozen spermatozoa Journal of Animal Science Supplement 173232 (Abstract 488)

Shannon P (1978) Factors affecting semen preservation and conception rates in cattle Journal of Reproduction and Fertility 54 519-527

Smith TT and Yanagimachi R (1989) Capacitation status of hamster spermatozoa in the oviduct at various times after mating Joumal of Reproduction and Fertility 86 255-261

Smith TT and Yanagimachi R (1991) Attachment and release of spermatozoa from the caudal isthmus of the hamster oviduct Journal of Reproduction and Fertility 91 567-573

Suzuki H, Foote RH and Farrell PB (1997) Computerized imaging and scanning electron microscope (SEM) analysis of co-cultured fresh and frozen bovine sperm lournal of Andrology 18 217-226

Tilbrook AJ and Pearce DT (1984) Losses of spermatozoa from the vagina of the ewe Animal Production in Australia 15759 (Abstract)

Tsien RY (1989) Fluorescent indicators of ion concentrations. In Fluorescence Microscopy of Living Cells in Culture. Part B.
Quantitative Fluorescence Microscopy - Imaging and Spectroscopy. Methods in Cell Biology Vol. 30 pp 127-156 Eds DL Taylor and Y-L Wang. Academic Press, New York

Wang WH, Abeydeera LR, Fraser LR and Niwa K (1995) Functional analysis using chlortetracycline fluorescence and in vitro fertilization of frozen-thawed ejaçuated boar spermatozoa incubated in a protein-free chemically defined medium Journal of Reproduction and Fertility 104 305-313

Watson PF (1995) Recent developments and concepts in the cryopreservation of spermatozoa and the assessment of their post-thawing function Reproduction, Fertility and Development 7 871-891

Watson PF (1996) Cooling of spermatozoa and fertilizing capacity. In Proceedings of the Third International Conference on Boar Semen Preservation, Mariensee, Germany Eds D Rath, LA Johnson and KF Weitze Reproduction in Domestic Animals 31 135-140

Windsor DP (1997) Mitochondrial function and ram sperm fertility Reproduction, Fertility and Development 9 279-284 